\title{
RELACIÓN DE LAS PROPIEDADES DEL SUELO CON VARIABLES BROMATOLÓGICAS DE PASTOS, EN UN SISTEMA LECHERO DE NARIÑO
}

\section{RELATIONSHIP BETWEEN SOIL PROPERTIES AND BROMATOLOGICAL VARIABLES GRASSES IN A DAIRY SYSTEM OF NARIÑO}

\author{
Gema Zambrano B. ; ; José Edmundo Apráez G. ${ }^{2}$; Jorge Fernando Navia E. ${ }^{3}$
}

Fecha de recepción: Abril 02 de 2014 Fecha de aceptación: Noviembre 10 de 2014

\begin{abstract}
RESUMEN
El presente estudio se realizó en la parte alto andina del departamento de Nariño y su objetivo principal fue evaluar la relación de las propiedades físicas y químicas del suelo con relación a la producción de biomasa y los análisis bromatológicas del monocultivo Lolium hybridum y la pradera en mezclas de Dactylis glomerata L., Holcus lannatus L. y Lolium sp. Los indicadores edáficos del monocultivo en las zonas altas, media y baja mostraron diferencias, pero no para las épocas analizadas (lluviosa-seca). En los pastos en mezclas, los resultados de las propiedades químicas, fueron estadísticamente diferentes. En las variables físicas de suelo, se presentó una textura FrancoArenosa, con buena estabilidad. En el monocultivo, la Da por zona mostró diferencias; mientras la Dr y porosidad total $(\mathrm{Pt})$ no las presentaron. Los resultados en la mezcla de pastos, por zona no mostró diferencias en todas las variables física de suelos (Dr, Da, y porosidad total). Sin embargo, por épocas si hubo diferencias en Dr y Pt. En el monocultivo, se observaron diferencias, en el periodo de recuperación (PR) y producción de biomasa (PB), tanto por época como por zona. No obstante, en la mezcla de pastos el PR fue diferente entre épocas, pero no se observaron diferencias por zona. En el análisis bromatológico se observaron diferencias únicamente por zona, para los componentes proteína cruda, fibra detergente neutro, fibra detergente ácido, calcio, fósforo, magnesio y nutrientes digestibles totales, tanto en la pradera en monocultivo como en mezcla. Las
\end{abstract}

1 Investigador, Zoot. M.Sc. Universidad de Nariño, Pasto, Nariño, Colombia, mitugema@hotmail.com

2 Profesor Asociado, Zoot. Ph.D. Universidad de Nariño, Pasto, Nariño, Colombia, eapraez@udenar.edu.co

3 Profesor Asociado, Ph.D. Universidad de Nariño, Pasto, Nariño, Colombia, jornavia@yahoo.com 
relaciones positivas de estas variables edáficas con la producción permitirían una alta relación con la recuperación de la pradera y el bienestar animal.

Palabras Clave: Dactylis glomerata L., Holcus lannatus L., Lolium sp, Lolium hybridum, sistema de producción animal, Trópico Alto de Nariño.

\begin{abstract}
The research was conducted at high Andean regions of the department of Nariño with the aim of evaluating the physical and chemical properties of soil and its relation to biomass production and bromatological analyses of Lolium hybridum monoculture and pasture mixtures of Dactylis glomerata L., Holcus lannatus L. y Lolium sp. Soil indicators for monoculture at high, medium, and low areas showed differences between these, but no differences were observed between seasons (wet-dry). The chemical properties were statistically different for the pasture mixtures. In regards to the physical properties, the soils showed a sandy-loam texture with good stability. In monoculture, the bulk density (BD) per area showed differences, while the relative density (RD) and total porosity (TP) did not present differences. The results for the mixed pastures perarea showed no differences in all of the physical soil variables (RD, $\mathrm{BD}$, and $\mathrm{TP}$ ) between areas; however, differences in RD and TP were found between seasons $(\mathrm{P}<0.05)$. In monoculture, differences in the recovery period $(\mathrm{RP})$ and biomass production $(\mathrm{PB})$ forboth seasons and areas were found. Nonetheless, in the pasture mixture, RP differed between seasons, but no differences were found per area. The bromatological analysis showed only differences between locations for monoculture as well as pasture mixtures, in regards to raw protein, neutral detergent fiber, acidic detergent fiber, calcium, phosphorus, magnesium, and total digestible nutrients. The positive relationships of these soil variables with production will allow a high relation between pasture recovery and animal wellbeing.
\end{abstract}

Keywords: Dactylis glomerata L., Holcus lannatus L., Lolium sp, Lolium hybridum, animal production system, High Tropic of Nariño.

\section{INTRODUCCIÓN}

Colombia es un país tropical de contrastes climáticos debido a su topografía y a su posición latitudinal, comprende cinco ecorregiones entre las que se encuentra la zona andina, con una extensión cercana al 30\% del total del territorio nacional, donde se asienta el 75\% de la población del país. En esta región se ubican las grandes ciudades, las cuales demandan continuamente altos volúmenes de alimentos, que se producen de manera intensiva, factor que ha causado deterioro de los suelos, el cual presenta contaminación causada, principalmente por el uso de agroquímicos y por las continuas labores tradicionales de mantenimiento de los cultivos (Viloria, 2007).

En el campo pecuario, la zona andina concentra el 52\% de las lecherías especializadas del país, por lo que actualmente la presión sobre este ecosistema se hace aún mayor. La producción de leche se ha incrementado, debido a la creciente demanda del mercado interno. Esta actividad, ha sido un factor de mitigación de la crisis que vive el sector agropecuario, dado que al igual que la producción de derivados lácteos, se ha constituido en una labor fundamental para la 
dinámica y recuperación del quehacer pecuario, aportando dentro del PIB agropecuario un 10\% durante los últimos años (MADR, 2008).

En el Trópico Alto de Nariño, la ganadería de leche tiene gran importancia social y económica, ya que de ella dependen directa e indirectamente millares de familias que obtienen sus ingresos de esta actividad. Las difíciles condiciones en que se encuentran las actuales producciones ganaderas de leche están relacionadas con la baja rentabilidad, resultado de factores ambientales, nutricionales, genéticos, sanitarios y sumado a la incertidumbre de enfrentar la globalización con leche de buena calidad a menor precio (Garcés et al., 2004). En este sentido, en Nariño no existe un modelo de producción que articule los componentes del sistema pecuario, con el fin de establecer las relaciones entre ellos $\mathrm{y}$ determinar los correctivos $\mathrm{u}$ orientaciones de manera integral en la ganadería.

En consecuencia, el objetivo de la investigación fue evaluar la relación entre las propiedades físicas y químicas del suelo, con la producción y calidad nutritiva de los pastos en monocultivo versus una pradera asociada, que permita contribuir con el mejoramiento de la ganadería especializada de leche en Nariño.

\section{MATERIALES Y MÉTODOS}

El trabajo de investigación, se llevó a cabo en la finca los Arrayanes, ubicada en la vereda $\mathrm{Cu}$ bijan Alto, del corregimiento de Catambuco, municipio de Pasto. Coordenadas geográficas $1^{\circ} 09^{\prime} 54,89^{\prime \prime}$ norte y $77^{\circ} 19^{\prime} 18,64^{\prime \prime}$ oeste. Esta finca, se caracteriza por su tradición desde hace más de 35 años, en producir leche. Cuenta con un área total de 30 hectáreas; altura promedio de 3000 msnm; temperatura media de $12{ }^{\circ} \mathrm{C}$ y el sistema de ordeño es mecánico $100 \%$. De acuerdo con el IGAC (2003), los suelos de la finca los Arraya- nes son andisoles, los cuales son muy representativos desde el punto de vista de la tipología, ya que integran el $11 \%$ de Hapludands, el 5\% de Melanudands y el conjunto de los Melanocryands, Placudands, Fulvudandsy Haplustands el 0,5\%.

Los tratamientos, se diseñaron con relación al tipo de pradera presente en la finca estudiada. Para este estudio las praderas seleccionadas fueron monocultivo (Tratamiento 1) constituido de pasto Sterling Lolium hybridum y el tratamiento 2 fue la pastura pasto Sterling Lolium hybridum Hausskn. con la mezcla de azul orchoro Dactylis glomerata L., Falsa poa Holcus slannatus L. y Raygrass Lolium sp. Los muestreos de suelos y de pastos, se realizaron en tres zonas alta, media y baja, teniendo en cuenta la pendiente del terreno y en dos épocas (lluvias y seca) del año 2012.

Para el muestreo de componente edáfico, fue necesario calcular el porcentaje de pendiente en cada lote, dicho cálculo se realizó con GPS map 60 CSx. De cada franja, se tomó tres submuestras de suelo, con anillos de $0,5 \mathrm{~cm}$ de diámetro. Los porcentajes de pendiente para la pradera en monocultivo Lolium hibridum L. fueron: zona alta $14 \%$; zona media $10 \%$ y zona baja $7 \%$ y para la pradera en mezclas Dactylis glomerata, Holcus lannatus L. y Lolium sp, fueron: zona alta $16 \%$; zona media $12 \%$ y zona baja $8 \%$.

Se analizaron las propiedades físicas textura, penetrabilidad, estabilidad de agregados, densidad real, densidad aparente y porosidad total. Las variables químicas $\mathrm{MO}, \mathrm{pH}, \mathrm{CIC}, \mathrm{P}, \mathrm{K}, \mathrm{Ca}$ y $\mathrm{Mg}$. Todas estas variables se analizaron, de acuerdo con los procedimientos descritos en el Laboratorio de Física de suelos y por el Manual de Métodos Químicos para el análisis de suelos (Unigarro et al., 2009).

Las variables evaluadas y las técnicas utilizadas, se resumen en la Tabla 1. 
Tabla 1. Variables y técnicas utilizadas para las propiedades físicas y químicas del suelo analizado (laboratorios especializados, Universidad de Nariño, 2012)

\begin{tabular}{|c|c|c|}
\hline Variables & Metodologías & Referencia \\
\hline \multicolumn{3}{|c|}{ Físicas } \\
\hline Densidad aparente & Volumen de cilindro conocido & IGAC, 2006 \\
\hline Densidad real & Picnómetro & IGAC, 2006 \\
\hline Textura & Hidrímetro Bouyoucos & IGAC, 2006 \\
\hline Penetrabilidad & Penetrógrafo & IGAC, 2006 \\
\hline Estabilidad de agregados & Yoder & IGAC, 2006 \\
\hline Porosidad total & $1-\mathrm{Da} / \mathrm{Dr})^{*} 100$ & IGAC, 2006 \\
\hline \multicolumn{3}{|c|}{ Químicas } \\
\hline Materia orgánica & Wakley-Black - Colorimetría & IGAC, 2006 \\
\hline $\mathrm{P}$ & Bray Kurtz colorimétrico & IGAC, 2006 \\
\hline $\mathrm{K}, \mathrm{Ca}, \mathrm{Mg}$ & $\begin{array}{l}\text { Acetato de amonio } 1 \mathrm{~N}, \mathrm{pH} 7 . \\
\text { Absorción atómica }\end{array}$ & IGAC, 2006 \\
\hline $\mathrm{pH}$ & $\begin{array}{l}\text { Potenciómetro relación suelo } \\
\text { agua } 1: 1\end{array}$ & IGAC, 2006 \\
\hline $\begin{array}{l}\text { Capacidad de Intercambio } \\
\text { Catiónico }\end{array}$ & $\begin{array}{l}\text { Acetato de amonio } 1 \mathrm{~N}, \mathrm{pH} 7 . \\
\text { Volumétrico }\end{array}$ & IGAC, 2006 \\
\hline
\end{tabular}

Para evaluar la productividad de las praderas, se procedió a ubicar las áreas, teniendo en cuenta la homogeneidad del forraje y se recolectaron tres muestras de cada pradera de pastos, para lo cual se tuvo en cuenta la altura, el color y densidad $(\mathrm{Kg} / \mathrm{m} 2)$ del forraje al momento del corte. En cada lote experimental se realizó un corte, el cual se hizo a $15 \mathrm{~cm}$ del piso, con el fin de determinar el periodo de recuperación. Posteriormente, se determinó mediante análisis bromatológico, en los laboratorios de la Universidad de Nariño, la calidad de los pastos, principalmente en componentes de proteína cruda, fibra detergente neutro, fibra detergente ácido, calcio, fósforo, magnesio y nutrientes digestibles totales (NDT), que expresa la forma energética del pasto. El cálculo de los NDT, se realizó teniendo en cuenta la energía digestible (ED) $/ 4,38) * 100$.
Dónde:

$\mathrm{ED}=((0,0504 *$ Proteína cruda $)+(0,02 *$ Fibra Cruda $)+$ $(0,077 *$ Extracto etéreo $)+(0,011 *$ Extracto libre de nitrógeno $)$

+ “ $\left(0,000377 *\right.$ Extracto libre de nitrógeno $\left.\left.{ }^{2}\right)\right)-152$

Se aplicó un diseño Switch back y un análisis de variancia (Programa Meg@lac, 2009). Finalmente, se correlacionaron entre sí todas las variables utilizando el paquete estadístico Statgraphics Centurion XV.II.

\section{RESULTADOS Y DISCUSIÓN}

\section{Componente edáfico de las praderas}

En la Tabla 2, con relación a las variables químicas del suelo del monocultivo (T1) Lolium 
hybridum, y del sistema pradera en mezcla Lolium hybridum, Dactylis glomerata, Holcus lannatus y Lolium sp. (T2) se presentaron diferencias significativas a altamente significativas en todas las variables $\mathrm{pH}$, materia orgánica, potasio, magnesio, calcio y capacidad de intercambio catiónico, por zona y época al (P>0,05), excepto la variable fósforo, donde no se presentaron diferencias por zona y época.

Lo anterior, permite afirmar que los contenidos de materia orgánica del suelo, fueron altos, tanto en la época y zona, respecto a las necesidades del pasto, las cuales oscilan entre $8-12 \%$, según lo reportado por el Instituto As Research Grasslands de Nueva Zelanda (2010). Este indicador, permite inferir que la pradera Lolium hybridum, cultivado en este suelo, presentará un perfil nutricional favorable para la alimentación de la ganadería de leche.

Respecto al $\mathrm{pH}$, el valor promedio por zona fueron similares, excepto en la zona baja $(P>0,05)$, donde se presentó menos acidez, en las dos épocas de estudio. La acidez de los suelos, permite la solubilidad de los nutrientes y su disponibilidad para el pasto. Este resultado, también se relacionó con el tipo de suelo (Franco-Arenoso), lo que confirma que el contenido de materia orgánica, que fluctuó entre $18-87 \%$ a $21,52 \%$, está condicionado por los niveles de acidez en los suelos (Bernal, 1994; Burbano y Cadena, 2009; Tapia y Rivera, 2010).

Para la capacidad de intercambio catiónico (CIC), se obtuvieron promedios similares $(\mathrm{P}>0,05)$, tanto para las épocas como por zona (Tab. 3). De acuerdo con Salamanca (1986), valores superiores a 20 Cmolc.kg ${ }^{-1} / 100 \mathrm{~g}$ indican una CIC alta, posiblemente debido a la alta cantidad de materia orgánica presente, ya que estas dos variables están altamente correlacionadas. Frente a esto, Gavilán (2004) sostiene que los materiales orgánicos presentan una elevada CIC y por tanto, una alta capacidad tampón frente a cambios rápidos en la disponibilidad de nutrientes y en el pH. En consecuencia, la CIC elevada constituyó un depósito de reservas para los nutrientes, lo cual favoreció el comportamiento productivo del pasto Lolium hybridum en este tipo de suelos.

Tabla 2. Probabilidad de la prueba F del análisis de varianza y desviación estándar de las variables químicas por época y zona del monocultivo Lolium hybridum Hausskn y pradera en mezclas de Dactylis glomerata, Holcus lannatus L. y Lolium sp.

\begin{tabular}{|c|c|c|c|c|c|c|c|c|}
\hline \multirow[b]{2}{*}{ Variables } & \multicolumn{4}{|c|}{ Probabilidad por zona } & \multicolumn{4}{|c|}{ Probabilidad por época } \\
\hline & $\begin{array}{l}\text { Lolium } \\
\text { hybridum } \\
\text { Hausskn }\end{array}$ & Sdt & $\begin{array}{l}\text { Dactylis } \\
\text { glomerata } \\
\text { L., Holcus } \\
\text { lannatus L. y } \\
\text { Lolium sp. }\end{array}$ & Sdt & $\begin{array}{c}\text { Lolium } \\
\text { hybridum } \\
\text { Hausskn }\end{array}$ & Sdt & $\begin{array}{c}\text { Dactylis } \\
\text { glomerata } \\
\text { L., Holcus } \\
\text { lannatus L. } \\
\text { y Lolium } \\
\text { sp. }\end{array}$ & Sdt \\
\hline $\mathrm{pH}$ & $0,00005^{* *}$ & 0,026 & 0,93 & 0,571 & $0,00005^{* *}$ & 0,002 & 0,93 & 0,001 \\
\hline $\mathrm{MO} \%$ & $0,00005^{\star *}$ & 0,020 & 0,935 & 0,716 & $0,00005^{\star *}$ & 0,004 & 0,91 & 0,014 \\
\hline $\mathrm{P}\left(\mathrm{mg} \cdot \mathrm{kg}^{-1}\right)$ & 0,161 & 0,032 & 0,714 & 0,018 & 0,185 & 0,001 & 0,10 & 0,058 \\
\hline $\mathrm{K}\left(\right.$ Cmolc.kg $\left.{ }^{-1} / 100 \mathrm{~g}\right)$ & $0,00005^{* *}$ & 1,731 & 0,934 & 0,475 & $0,00005^{* *}$ & 0,001 & 0,86 & 0,003 \\
\hline Ca (Cmolc.kg $\left.{ }^{-1} / 100 g\right)$ & $0,00005^{* *}$ & 0,192 & 0,923 & 0,344 & $0,00005^{* *}$ & 0,020 & 0,98 & 0,002 \\
\hline Mg (Cmolc.kg -1/100g) & $0,00005^{* *}$ & 0,986 & 0,952 & 0,496 & $0,00005^{* *}$ & 0,007 & 0,98 & 0,002 \\
\hline CIC (Cmolc.kg $\left.{ }^{-1} / 100 \mathrm{~g}\right)$ & $0,00005^{* *}$ & 0,543 & 0,950 & 0,508 & $0,00005^{* *}$ & 0,012 & 0,97 & 0,026 \\
\hline
\end{tabular}

** Significativo $(\mathrm{P}<0,05)$; Época 1. Lluviosa; Época 2. Seca 
En relación a los minerales $\mathrm{P}, \mathrm{K}, \mathrm{Ca}$ y $\mathrm{Mg}$, presentaron promedios similares $(\mathrm{P}>0,05)$, en las épocas y zonas de estudio. En el caso del $\mathrm{Ca}$, los valores encontrados fueron altos, 12,38, 9,38 y 12,31 para la época lluviosa y para cada zona, respectivamente. Igualmente, en la época seca, en las mismas zonas fueron 12,35, 9,37 y 12,21, respectivamente. No obstante, el contenido de Ca en el suelo, está condicionado por el grado de acidez del mismo. El contenido de este mineral en el pasto fue alto, lo que permite suponer que quizá hubo otros elementos que estuvieron en juego en la movilización de este elemento y su asimilación por el pasto (Donald, 2007). Los resultados medios de $\mathrm{K}$, fueron altos, lo que confirma lo manifestado por Urbano (1992), quien sostiene que el $\mathrm{K}$ que se encuentra combinado con la materia orgánica, puede ser liberado en forma de $\mathrm{K}^{+}$mediante el proceso de mineralización de la misma, mediado por la población microbiana.

Los valores de $\mathrm{Mg}$ (Tab. 3) son considerados altos, lo que permite que, la disponibilidad del mismo para la planta se encuentre en buena cantidad (Donald, 2007). Según Parra (2003), el Ca y $\mathrm{Mg}$ tienen un comportamiento muy similar en el suelo y estos elementos son los que se encuen- tran generalmente en niveles más altos. La concentración y la disponibilidad de ambos, están controladas generalmente por la CIC de manera directa. Por lo tanto, al tener una CIC alta, permite una mayor disponibilidad de nutrientes disueltos en el suelo.

La cantidad media de fósforo obtenido en esta investigación fue baja y no presento diferencias por zona y época (Tab. 3). Sin embargo, la relación 2:1 de Ca-P, es apropiada, ya que los suelos estudiados son de origen volcánico, lo que contribuye a que sean ricos en dichos elementos, además, los niveles altos de materia orgánica favorecen la mineralización que esta realiza (Tapia y Rivera, 2010).

Durán (2003), afirma que en clima frío valores superiores al 10\% de MO se consideran altos, por tanto, los resultados obtenidos por zona, resultaron superiores y el análisis de varianza reveló diferencias significativas $(\mathrm{P}<0,05)$. Por el contrario, no se observaron diferencias significativas $(\mathrm{P}>0,05)$, para el análisis por época. El alto contenido de $\mathrm{MO}$, permite que existan altos contenidos de $\mathrm{Ca}$, $\mathrm{K}$ y $\mathrm{Mg}$ y por ende una buena $\mathrm{CIC}$, tanto por época como por zona.

Tabla 3. Prueba de medios de las variables químicas por época y zona del monocultivo Lolium hybridum Hausskn y pradera en mezclas de Dactylis glomerata L., Holcus lannatus L. y Lolium sp.

\begin{tabular}{|c|c|c|c|c|c|c|c|c|c|c|c|c|}
\hline \multirow{3}{*}{ Variable } & \multicolumn{6}{|c|}{ Lolium hybridum Hausskn } & \multicolumn{6}{|c|}{ Dactylis glomerata L., Holcus lannatus L. y Lolium sp. } \\
\hline & \multicolumn{3}{|c|}{ Época 1} & \multicolumn{3}{|c|}{ Época 2} & \multicolumn{3}{|c|}{ Época 1} & \multicolumn{3}{|c|}{ Época 2} \\
\hline & $\mathrm{ZA}$ & $\mathrm{ZM}$ & $\mathrm{ZB}$ & $\mathrm{ZA}$ & $\mathrm{ZM}$ & $\mathrm{ZB}$ & $\mathrm{ZA}$ & $\mathrm{ZM}$ & $\mathrm{ZB}$ & $\mathrm{ZA}$ & ZM & $\mathrm{ZB}$ \\
\hline $\mathrm{MO} \%$ & $20,76 a$ & $20,44 a$ & $19,3 b$ & $20,7 \mathrm{a}$ & $20,45 a$ & $19,2 b$ & $21,52 \mathrm{a}$ & $18,90 \mathrm{~b}$ & $20,44 a$ & $21,45 a$ & $18,87 \mathrm{~b}$ & $20,42 \mathrm{a}$ \\
\hline $\mathrm{pH}$ & $5,82 b$ & $5,8 b$ & $6,17 \mathrm{a}$ & $5,8 b$ & $5,7 \mathrm{~b}$ & $6,18 \mathrm{a}$ & $5,90 \mathrm{~b}$ & $6,19 a$ & $6,11 a$ & $5,9 b$ & $6,19 a$ & $6,10 \mathrm{a}$ \\
\hline CIC (Cmolc.kg $\left.{ }^{-1} / 100 \mathrm{~g}\right)$ & $16,94 b$ & $14,27 \mathrm{c}$ & $18,37 \mathrm{a}$ & $16,9 b$ & $14,28 \mathrm{c}$ & $18,29 a$ & $23,46 b$ & $20,62 c$ & $33,41 \mathrm{a}$ & $23,39 b$ & $20,55 c$ & $33,39 a$ \\
\hline P (mg.kg $\left.{ }^{-1}\right)$ & $5,91 \mathrm{a}$ & $6,02 a$ & $5,98 \mathrm{a}$ & $5,93 a$ & $5,95 a$ & $5,99 a$ & $5,81 \mathrm{a}$ & $6,01 \mathrm{a}$ & $5,95 \mathrm{a}$ & $5,99 a$ & $5,97 a$ & $6,06 a$ \\
\hline K (Cmolc.kg $\left.{ }^{-1} / 100 \mathrm{~g}\right)$ & $1,19 \mathrm{c}$ & $1,58 b$ & $2,35 \mathrm{a}$ & $1,17 \mathrm{c}$ & $1,6 b$ & $2,41 \mathrm{a}$ & $2,26 a$ & $1,58 b$ & $1,61 b$ & $2,23 a$ & $1,55 b$ & $1,62 b$ \\
\hline $\mathrm{Ca}\left(\mathrm{Cmolc}_{\mathrm{kg}}{ }^{-1} / 100 \mathrm{~g}\right)$ & $12,38 \mathrm{a}$ & $9,38 b$ & $12,31 \mathrm{a}$ & $12,35 a$ & $9,37 \mathrm{~b}$ & $12,21 \mathrm{a}$ & $15,17 \mathrm{~b}$ & $14,6 b$ & $21,8 \mathrm{a}$ & $15,14 b$ & $14,59 b$ & $21,7 \mathrm{a}$ \\
\hline $\operatorname{Mg}\left(\right.$ Cmolc.kg $\left.{ }^{-1} / 100 \mathrm{~g}\right)$ & $3,37 b$ & $3,3 b$ & $3,7 \mathrm{a}$ & $3,38 b$ & $3,30 \mathrm{~b}$ & $3,67^{\mathrm{a}}$ & $6,02 b$ & $4,44 \mathrm{c}$ & $10,0 \mathrm{a}$ & $6,01 b$ & $4,41 \mathrm{c}$ & $9,99 a$ \\
\hline
\end{tabular}

ZA: Zona Alta; ZM: Zona Media; ZB: Zona Baja; Época 1. Lluviosa; Época 2. Seca 
Por otro lado, los contenidos de MO están explicados por la altura y temperatura de la zona de estudio, esto coincide con Charry (1987), quien menciona que a mayor altitud la materia orgánica se incrementa, debido al lento proceso de mineralización de la misma, por la baja actividad de los microorganismos del suelo. Las anteriores variables químicas, mostraron diferencias altamente significativas $(\mathrm{P}<0,05)$ por zona. Los análisis de varianza por época, para las variables mencionadas no mostraron diferencias significativas $(\mathrm{P}>0,05)($ Tab. 2$)$.

Bernal (2003), afirma que el rango óptimo de $\mathrm{pH}$ para el crecimiento de la mayoría de las pasturas, oscila entre 5.5 y 6.5 . En este sentido, el valor promedio obtenido en esta investigación, por zona y época, se encontró en dicho rango. Según estos resultados, los suelos se pueden catalogar como ligeramente ácidos, que confirman la condición de los suelos en Nariño, especialmente los derivados de cenizas volcánicas. El análisis estadístico por zona de estudio, resultó estadísticamente significativa $(\mathrm{P}<0,05)$. Sin embargo, el análisis por época no reveló diferencias $(\mathrm{P}>0,05)$.
Respecto a los análisis de las propiedades físicas del suelo, indican que la textura de los suelos corresponden a Franco-Arenosos, es decir, que la distribución de las partículas en el suelo son en su mayoría de 0,05 a $2.00 \mathrm{~mm}$, contiene alrededor del $60-70 \%$ de arena y $20 \%$ de arcilla. Estos resultados concuerdan con los reportados por Burbano y Cadena (2009) y Beltrán y Benavides (2009), en estudios realizados en Guachucal, departamento de Nariño.

En cuanto a la estabilidad de agregados, se puede mencionar que los suelos fueron estables, ya que el Diámetro Medio Ponderado (DMP), osciló entre 3,0 y 5,0. Es decir, que dichos suelos, tienen una adecuada estructura, que permiten no ser susceptibles a erosión.

El análisis de varianza por zona del monocultivo Lolium hybridum, resultó altamente significativo $(\mathrm{P}<0,05)$ para densidad aparente $(\mathrm{Da})$; mientras que para densidad real (Dr) y la porosidad total no se evidenciaron diferencias significativas $(\mathrm{P}>0,05)$. Los análisis estadísticos por época, no fueron significativos $(\mathrm{P}>0,05)$ para ninguna de las variables mencionadas anteriormente (Tab. 4).

Tabla 4. Probabilidad y desviación estándar de las variables físicas por época y zona del monocultivo Lolium hybridum Hausskn y la pradera en mezcla de

Dactylis glomerata L., Holcus lannatus L.y Lolium sp.

\begin{tabular}{ccccccccc}
\hline & \multicolumn{3}{c}{ Probabilidad entre zonas } & \multicolumn{3}{c}{ Probabilidad entre épocas } \\
\cline { 2 - 9 } Variables & $\begin{array}{c}\text { Lolium } \\
\text { hybridum } \\
\text { Hausskn }\end{array}$ & Sdt & $\begin{array}{c}\text { Dactylis } \\
\text { glomerata L., } \\
\text { Holcus lannatus } \\
\text { L.y Lolium sp }\end{array}$ & Sdt & $\begin{array}{c}\text { Lolium } \\
\text { hybridum }\end{array}$ & Sdt & $\begin{array}{c}\text { Dactylis } \\
\text { glomerata } \\
\text { L., Holcus } \\
\text { lannatus L.y } \\
\text { Lolium sp }\end{array}$ & Sdt \\
\hline $\mathrm{Da}(\mathrm{g} / \mathrm{cc})$ & $0,014^{* *}$ & 0,004 & 0,87 & 0,001 & 0,65 & 0,002 & 0,90 & 0,001 \\
$\mathrm{Dr}(\mathrm{g} / \mathrm{cc})$ & 0,17 & 0,045 & 0,80 & 0,009 & 0,21 & 0,041 & $0,0025^{* *}$ & 0,336 \\
Porosidad\% & 0,73 & 0,217 & 0,40 & 0,820 & 0,52 & 0,273 & $0,0005^{* *}$ & 0,748 \\
\hline
\end{tabular}

** Significativo $(\mathrm{P}<0,05)$; Época 1. Lluviosa; Época 2. Seca 
Los resultados de la Tabla 5, indican que la Da tuvo valores similares entre época y zona. Estas cifras, están directamente relacionadas con los altos contenidos de $\mathrm{MO}$ en el suelo, el cual permite que exista mayor retención de agua y por ende regula la nutrición de la planta, al estimular el sistema radicular. Además, el tipo de suelos Franco-Arenosos, la estabilidad del mismo, combinado con el porcentaje de porosidad favorecen la nutrición de la planta, al permitir que las raíces profundicen adecuadamente (León y Zambrano, 2008; Apráez et al., 2012).

Al respecto, Thompson (1988) manifiesta que la MO hace disminuir la densidad aparente, ya que por equivalencia de volumen es mucho más ligera que la materia mineral e incrementa la estabilidad de los agregados del suelo, siendo este segundo efecto el más importante en la mayoría de los suelos. Según Jaramillo (2004) y Burbano y Cadena (2009), coinciden en que la densidad real varía entre 2,6 a $2.75 \mathrm{~g} / \mathrm{cc}$ en todos los suelos agrícolas.

Los resultados de los mencionados autores son similares con los indicados en la Tabla 4, donde se presentan los valores de densidad real, los cuales mostraron diferencias entre épocas, observa cifras inferiores en época seca. Igualmente, ocurre al analizar los promedios por zona. Valores similares, fueron encontrados por Beltrán y Benavides (2009), quienes reportan 2,34 $\mathrm{g} / \mathrm{cc}$ de densidad real, en suelos del municipio de Guachucal del departamento de Nariño.

Igualmente, Herrera et al. (1989) encontraron una densidad real de $2,31 \mathrm{~g} / \mathrm{cc}$, en un suelo bajo labranza mínima con dos pases de rastrillo de disco. Además, mencionan que los valores de densidad real son difíciles de alterar, ya que esta variable se relaciona con las características mineralógicas del material parental. Sin embar- go, la erosión y excesiva compactación puede lograrlo en el largo plazo.

Los valores de porosidad que oscilaron entre 66 a $70 \%$, son excelentes para el monocultivo Lolium hybridum. Los valores están relacionados con los contenidos de materia orgánica (Tapia y Rivera, 2010). Mila (2001), Soriano y Pons (2004), coinciden en que la porosidad se relaciona directamente con la retención y movimiento del agua en el perfil del suelo. Es una propiedad física esencial ya que la aireación y transporte de oxígeno al sistema radicular de las plantas garantizan la facilidad con que las raíces pueden anclar y sostenerse en el suelo y permitir así la rápida absorción de nutrientes de la solución del suelo. Los resultados obtenidos, favorecieron el crecimiento y desarrollo del pasto neozelandés, ya que le permitieron que la planta disponga de los minerales presentes en el suelo y por ende alcanzar mayor productividad de biomasa (Tab. 5).

Para el caso de la pradera en mezclas Dactylis glomerata, Holcus lannatus L., Lolium sp, no se encontraron diferencias $(\mathrm{P}>0,05)$ por zona para las variables $\mathrm{Da}$, Dr y porosidad total. Sin embargo, se encontraron diferencias altamente significativas $(\mathrm{P}<0,05)$ por época para las características Dr y porosidad total; mientras que la $\mathrm{Da}$, no fue significativa $(\mathrm{P}>0,05)$.

En la Tabla 5, se indican los valores promedio de las características físicas del suelo $\mathrm{Da}, \mathrm{Dr}$ y porosidad total, por época y zona de estudio, para la pradera en mezclas. Al evaluar estas características en conjunto, se puede afirmar que los suelos de la pradera en mezclas, presentaron buenas condiciones físicas, para la producción de Dactylis glomerata, Holcus lannatus L. y Lolium sp. 
Tabla 5. Prueba de medios de las variables físicas por época y zona del monocultivo Lolium hybridum Hausskn y la pradera en mezcla de Dactylis glomerata L., Holcus lannatus L. y Lolium sp.

\begin{tabular}{|c|c|c|c|c|c|c|c|c|c|c|c|c|}
\hline \multirow{3}{*}{ Variable } & \multicolumn{6}{|c|}{$\begin{array}{l}\text { Lolium hybridum } \\
\text { Hausskn }\end{array}$} & \multicolumn{6}{|c|}{$\begin{array}{c}\text { Dactylis glomerata L., Holcus lannatus L. } \\
\text { y Lolium sp. }\end{array}$} \\
\hline & \multicolumn{3}{|c|}{ Época 1} & \multicolumn{3}{|c|}{ Época 2} & \multicolumn{3}{|c|}{ Época 1} & \multicolumn{3}{|c|}{ Época 2} \\
\hline & $\mathbf{Z A}$ & ZM & ZB & $\mathbf{Z A}$ & $\mathbf{Z M}$ & ZB & $\mathbf{Z A}$ & $\mathbf{Z M}$ & ZB & $\mathbf{Z A}$ & $\mathbf{Z M}$ & ZB \\
\hline $\mathrm{Da}(\mathrm{g} / \mathrm{cc})$ & 0,70 & 0,70 & 0,66 & 0,68 & 0,68 & 0,71 & 0,70 & 0,69 & 0,67 & 0,70 & 0,70 & 0,66 \\
\hline $\operatorname{Dr}(\mathrm{g} / \mathrm{cc})$ & 2,25 & 2,28 & 2,32 & 2,06 & 2,13 & 2,08 & 2,24 & 2,21 & 2,07 & 2,17 & 2,06 & 2,10 \\
\hline Porosidad\% & 68,3 & 69,9 & 70,9 & 66,0 & 68,0 & 66,3 & 68,0 & 68,0 & 67,0 & 67,0 & 66,0 & 68,0 \\
\hline
\end{tabular}

ZA: Zona Alta; ZM: Zona Media; ZB: Zona Baja; Época 1. Lluviosa; Época 2. Seca

\section{Componente productivo de las praderas}

El análisis de varianza para el periodo de recuperación (días), tanto por zonas y por épocas fueron significativos para el monocultivo Lolium hybridum. (Tab. 6). Los valores oscilaron entre 26 a 32 días (Tab. 7). Dichos resultados son similares con los reportados en investigaciones realizadas por Apráez et al. (2012); Mera y Ruales (2007). Respecto a la producción de biomasa $\left(\mathrm{Kg} / \mathrm{m}^{2}\right)$, se encontraron valores que fluctuaron entre 4,4 a 4,8, con diferencias altamente significativas $(P<0,05)$, tanto entre épo- cas como entre zonas. Estos resultados, son concordantes por los citados en la ficha técnica del Instituto AsResearch Grasslands, de Nueva Zelanda (2010).

Tal como se presenta, en la Tabla 6, para el periodo de recuperación (días), la pradera en mezcla de gramíneas, presentó diferencias significativas $(P<0,05)$ entre épocas, pero no se observaron diferencias $(P>0,05)$ por zonas. Por otro lado, la producción de biomasa reveló diferencias significativas $(\mathrm{P}<0,05)$ por zona, más no por época $(\mathrm{P}>0,05)$.

Tabla 6. Probabilidad y desviación estándar de las características productivas del monocultivo Lolium hybridum y pradera en mezclas Dactylis glomerata L., Holcus lannatus y Lolium sp.

\begin{tabular}{|c|c|c|c|c|c|c|c|c|}
\hline \multirow[b]{2}{*}{ Variables } & \multicolumn{4}{|c|}{ Probabilidad entre zonas } & \multicolumn{4}{|c|}{ Probabilidad entre épocas } \\
\hline & $\begin{array}{c}\text { Lolium } \\
\text { hybridum } \\
\text { Hausskn }\end{array}$ & Sdt & $\begin{array}{l}\text { Dactylis } \\
\text { glomerata } \\
\text { L., Holcus } \\
\text { lannatus L. y } \\
\text { Lolium sp }\end{array}$ & Sdt & $\begin{array}{c}\text { Lolium } \\
\text { hybridum }\end{array}$ & Sdt & $\begin{array}{l}\text { Dactylis } \\
\text { glomerata } \\
\text { L., Holcus } \\
\text { lannatus L. y } \\
\text { Lolium sp }\end{array}$ & Sdt \\
\hline $\begin{array}{l}\text { Periodo de } \\
\text { recuperación } \\
\text { (días) }\end{array}$ & $0,0112 * *$ & 0248 & 0,1918 & 0,180 & $0,00005^{* *}$ & 0,156 & $0,00005^{* *}$ & 0,338 \\
\hline $\begin{array}{c}\text { Biomasa }(\mathrm{Kg} / \\
\left.\mathrm{m}^{2}\right)\end{array}$ & $0,0049 * *$ & 0,981 & $0,00005^{* *}$ & 0,622 & $0,00005^{* *}$ & 0,512 & 0,1541 & 0,083 \\
\hline
\end{tabular}

** Significativo $(\mathrm{P}<0.05)$; Época 1. Lluviosa; Época 2. Seca 
Tabla 7. Características productivas del monocultivo Lolium hybridum Hausskn y pradera en mezclas Dactylis glomerata L., Holcus lannatus L. y Lolium sp.

\begin{tabular}{|c|c|c|c|c|c|c|c|c|c|c|c|c|}
\hline \multirow{3}{*}{ Variable } & \multicolumn{6}{|c|}{ Lolium hybridum Hausskn } & \multicolumn{6}{|c|}{$\begin{array}{l}\text { Dactylis glomerata L., Holcus } \\
\text { lannatus L. y Lolium sp }\end{array}$} \\
\hline & \multicolumn{3}{|c|}{ Época 1} & \multicolumn{3}{|c|}{ Época 2} & \multicolumn{3}{|c|}{ Época 1} & \multicolumn{3}{|c|}{ Época 2} \\
\hline & $\mathbf{Z A}$ & Z M & $\mathbf{Z B}$ & $\mathbf{Z A}$ & $\mathbf{Z M}$ & $\mathbf{Z B}$ & $\mathbf{Z A}$ & $\mathbf{Z M}$ & ZB & $\mathbf{Z A}$ & $\mathbf{Z M}$ & ZB \\
\hline $\begin{array}{c}\text { Periodo de } \\
\text { recuperación (días) }\end{array}$ & $26 a$ & $26 \mathrm{a}$ & $27 \mathrm{a}$ & $30 \mathrm{a}$ & $30 \mathrm{a}$ & $32 \mathrm{a}$ & $45 \mathrm{a}$ & $46 a$ & $52 b$ & $55 \mathrm{a}$ & $56 \mathrm{a}$ & $60 \mathrm{~b}$ \\
\hline $\operatorname{Biomasa}\left(\mathrm{Kg} / \mathrm{m}^{2}\right)$ & $4,8 \mathrm{a}$ & $4,7 \mathrm{a}$ & $4,5 b$ & $4,7 \mathrm{a}$ & $4,7 \mathrm{a}$ & $4,4 b$ & $5,8 \mathrm{a}$ & $5,7 \mathrm{a}$ & $5,4 b$ & $5,3 a$ & $5,1 \mathrm{a}$ & $4,9 b$ \\
\hline
\end{tabular}

ZA: Zona Alta; ZM: Zona Media; ZB: Zona Baja

Los anteriores resultados señalados en la Tabla 7, indican que tanto en el monocultivo Lolium hybridum como la pradera en mezcla Dactylis glomerata, Holcus lannatus L. y Lolium sp. en la época lluviosa, tuvieron una mayor producción de biomasa y por tanto se puede incrementar la carga animal. En época seca, hubo una disminución leve de la producción de pasto, como consecuencias del buen manejo de las praderas en la finca los Arrayanes.

De acuerdo con Jojoa y Silva (2009); Beltrán y Benavides, (2009) y, Burbano y Cadena, (2009), en investigaciones realizadas en el trópico alto de Nariño, la producción de biomasa está directamente relacionada con las propiedades químicas y físicas del suelo, ya que la interacción entre las mismas benefician el crecimiento y desarrollo de la planta.

Otros factores determinantes, fueron los relacionados con la precipitación y la humedad de los suelos, la cual estuvo influenciada por la provisión de riego en la finca. Todos estos componentes, permitieron mantener la similitud del periodo de recuperación y por ende la disponibilidad de biomasa verde durante los dos periodos.

\section{Componente bromatológico de las praderas}

El análisis estadístico del monocultivo Lolium hybridum, presentó diferencias significativas $(\mathrm{P}<0,05)$ por zona, para las variables productivas como proteína, fibra detergente neutro (FDN), fibra detergente ácido (FDA), calcio $(\mathrm{Ca})$, fósforo $(\mathrm{P})$, magnesio $(\mathrm{Mg})$ y nutrientes digestibles totales (NDT). No obstante, no se presentó $(\mathrm{P}>0,05)$ por época de muestreo para las mismas variables. El contenido de proteína (Tab. 8) fue menor en la zona baja $(24,5 \%)$; mientras que en la zona media y alta fueron de $26,2 \%$ y $25,01 \%$, respectivamente. Estos resultados indican que el mayor contenido proteico del pasto, se obtuvo en la zona media. Por otro lado, estos contenidos nutricionales, pudieron estar condicionados por el manejo, el cual estuvo relacionado con la utilización continua de biofertilizantes en la finca. Además, de otros factores como la similitud de las propiedades físicas y químicas del suelo, las cuales permiten mantener la composición nutritiva del pasto. 
Tabla 8. Probabilidad y desviación estándar del análisis bromatológico del monocultivo Lolium hybridum Hausskn y la pradera en mezcla Dactylis glomerata L., Holcus lannatus L. y Lolium sp (\%BS)

\begin{tabular}{|c|c|c|c|c|c|c|c|c|}
\hline \multirow[b]{2}{*}{ Variables } & \multicolumn{4}{|c|}{ Probabilidad entre zonas } & \multicolumn{4}{|c|}{ Probabilidad entre épocas } \\
\hline & $\begin{array}{c}\text { Lolium } \\
\text { hybridum } \\
\text { Hausskn }\end{array}$ & Sdt & $\begin{array}{c}\text { Dactylis } \\
\text { glomerata } \\
\text { L., Holcu } \\
\text { slannatus L. } \\
\text { y Lolium sp }\end{array}$ & Sdt & $\begin{array}{c}\text { Lolium } \\
\text { hybridum } \\
\text { Hausskn }\end{array}$ & Sdt & $\begin{array}{c}\text { Dactylis } \\
\text { glomerata } \\
\text { L., Holcus } \\
\text { lannatus L. y } \\
\text { Lolium sp }\end{array}$ & Sdt \\
\hline Proteína & $0,0005 * *$ & 0,121 & $0,0005^{* *}$ & 0,913 & 0,342 & 0,537 & 0,440 & 0,336 \\
\hline FDN & $0,0005^{* *}$ & 0,243 & $0,0005^{* *}$ & 0,252 & 0,312 & 0,148 & 0,561 & 0,248 \\
\hline FDA & $0,0005^{* *}$ & 0,156 & $0,0005^{* *}$ & 0,172 & 0,410 & 0,101 & 0,610 & 0,121 \\
\hline $\mathrm{Ca}$ & $0,0005 * *$ & 0,014 & $0,0005 * *$ & 0,015 & 0,340 & 0,107 & 0,112 & 0,131 \\
\hline $\mathrm{P}$ & $0,0005^{* *}$ & 0,003 & $0,0005^{* *}$ & 0,002 & 0,231 & 0,006 & 0,113 & 0,024 \\
\hline $\mathrm{Mg}$ & $0,0005^{* *}$ & 0,002 & $0,0005^{* *}$ & 0,008 & 0,123 & 0,001 & 0,212 & 0,036 \\
\hline NDT & $0.0005^{* *}$ & 0.175 & $0,0005^{* *}$ & 0,160 & 0,361 & 0,941 & 0,312 & 0,821 \\
\hline
\end{tabular}

** Significativo $(\mathrm{P}<0,05)$; Época 1. Lluviosa; Época 2. Seca

Los bajos valores de FDA, se traducen en un alto contenido de hemicelulosa, carbohidrato de mayor solubilidad y fuente energética fácilmente aprovechable. Estos resultados, son el reflejo de la edad de corte, la fertilidad del suelo, la distribución uniforme de las lluvias principalmente. Al respecto, Bernal (2003), afirma que los porcentajes de FDA y FDN, pueden variar significativamente de acuerdo con la época de corte, estación del año, fertilización y las condiciones físicas y químicas que le proveen los suelos al pasto.

El contenido de minerales fue similar $(\mathrm{P}>0,05)$ en las tres zonas, notándose una relación 2:1 entre Ca y $\mathrm{P}$, que implica un manejo cuidadoso en la provisión de este pasto, en especial si este se suministra a animales con altas producciones. Esta relación positiva, favorece el crecimiento y mantenimiento del tejido esquelético, la actividad muscular y nerviosa, la coagulación sanguínea y la síntesis de la leche (Castro et al. 2008, Weiss, 2000).
McDowell et al., (1997), afirman que el contenido mineral de las plantas varía según la fertilidad del suelo, el clima, la especie de la planta, su estado de madurez, rendimiento y manejo. Según la NRC (1989) y Sánchez (2000), los requerimientos de magnesio para las vacas lactantes oscilan entre $0,18 \%$ y $0,21 \%$. Por lo tanto, los resultados obtenidos por zona resultaron bajos, lo que implica que pueden presentarse hipomagnesemia o tetania.

Finalmente los valores promedio de energía, indican que el valor más alto fue el de la zona media (66 \% de NDT). No obstante, valores inferiores se observaron en la zona alta y baja, lo que conlleva a sugerir que los animales de alta producción deban suplementarse energéticamente cuando sea este el alimento base de su dieta.

El análisis bromatológico de la pradera en mezclas Dactylis glomerata, Holcus lannatus y Lolium sp, reveló diferencias altamente significativas $(\mathrm{P}<0,05)$ para las variables proteína, Fi- 
bra Detergente Neutro (FDN), Fibra Detergente Ácido (FDA), Calcio (Ca), Fósforo (P), Magnesio (Mg) y Nutrientes Digestibles Totales (NDT), cuando se analizó cada variable por zona. No se presentaron diferencias $(\mathrm{P}>0,05)$ por época de muestreo para las mismas variables.

En la Tabla 9, se presentan los niveles de proteína encontrados en la zona alta, media y baja correspondientes a 22,8, 23,4 y 21,4, respectivamente. Para la mezcla Dactylis glomerata, Holcus lannatus y Lolium sp. se consideran normales y pueden obedecer a la edad en que fue cortado el pasto y al manejo dado al cultivo en la finca. Esto permite que sea más palatable para el ganado (Rodas, 2007).

Al respecto, Pirela (2007), sostiene que el contenido proteico en gramíneas tropicales es relativamente alto en los estadios iníciales de crecimiento, para luego caer marcadamente hasta antes de la floración. Esta afirmación la confirman estudios realizados en el departamento de Nariño por Beltrán y Benavides, 2009; Burbano y Cadena, 2009; Tapia y Rivera, 2010.
Los valores obtenidos de FDN en las tres zonas (Tab. 9), demuestran que este componente se encuentra dentro de los parámetros normales (Bernal, 2003).

Los resultados bajos de FDA en las tres zonas de estudio, repercutieron en un alto contenido de hemicelulosa, carbohidrato de alta solubilidad y fuente de fibra aprovechable. Estos resultados se debieron probablemente a la edad de corte y a la fertilidad del suelo (Molina et al., 1982).

Respecto a los minerales como el Calcio (Ca), presentó un rango menor en la zona baja; mientras que en la zona alta y media fueron mayores. El P registró valores similares, excepto en la zona media, donde fue menor. Sin embargo, la relación $\mathrm{Ca}: \mathrm{P}$, se considera apropiada para vacas en producción. Además, estos resultados son producto de la mineralización de la materia orgánica (Sánchez, 2007). Los contenidos de $\mathrm{Mg}$, fueron equivalentes, es decir, este mineral se encuentra en cantidad favorable para las vacas en pastoreo.

Tabla 9. Análisis bromatológico del monocultivo Lolium hybridum Hausskn y la pradera en mezcla Dactylis glomerata L., Holcus lannatus L.y Lolium sp (\%BS)

\begin{tabular}{|c|c|c|c|c|c|c|}
\hline \multirow[t]{2}{*}{ Variable } & \multicolumn{3}{|c|}{ Lolium hybridum Hausskn } & \multicolumn{3}{|c|}{$\begin{array}{c}\text { Dactylis glomerata L., Holcus } \\
\text { lannatus L. y Lolium sp }\end{array}$} \\
\hline & ZA & $\mathrm{ZM}$ & ZB & $\mathrm{ZA}$ & $\mathrm{ZM}$ & ZB \\
\hline Proteína & 25,01 & 26,2 & 24,5 & 22,8 & 23,4 & 21,4 \\
\hline FDN & 50,2 & 51,1 & 50,5 & 57,6 & 57,8 & 54,8 \\
\hline FDA & 25,3 & 25,4 & 24,7 & 26,5 & 26,2 & 26,5 \\
\hline Calcio & 0,21 & 0,26 & 0,19 & 0,58 & 0,63 & 0,51 \\
\hline Fósforo & 0,30 & 0,29 & 0,32 & 0,33 & 0,30 & 0,34 \\
\hline Magnesio & 0,13 & 0,16 & 0,14 & 0,24 & 0,26 & 0,24 \\
\hline NDT & 64,0 & 66,0 & 64,0 & 62,0 & 63,0 & 60,0 \\
\hline
\end{tabular}

ZA: Zona Alta; ZM: Zona Media; ZB: Zona Baja 
Los NDT, se consideran medios. Resultados que pudieron deberse principalmente a los contenidos de nitrógeno en los suelos, que le permitió a la planta tener mejor reservas energéticas (Benítez, 1983; Estrada, 2002). Por lo tanto, esta mezcla de pastos podría presentar tendencias a satisfacer los requerimientos en vacas lecheras.

A continuación, en la Tabla 10 se presentan las correlaciones más relevantes, entre las variables del suelo - planta.

Tal como se aprecia en la Tabla 6, se observó alta correlación +1 entre el contenido de proteína del pasto con el $\mathrm{pH}$ de suelo. Lo que permite explicar el papel de $\mathrm{pH}$ en la solubilidad del $\mathrm{N}$ del suelo y su consecuente aprovechamiento por la planta. También pudo estar relacionado con el tipo de suelo (Franco-Arenoso), ya que por el tamaño de sus partículas permite obtener mejores condiciones para el cultivo de pastos.

Igualmente, la proteína del pasto tuvo una alta correlación con el contenido de materia orgánica $(\mathrm{MO})$ y potasio $(\mathrm{K})$ de los suelos en estudio. Estos resultados, permiten afirmar que a mayor contenido de $\mathrm{MO}$ en los suelos, se obtiene mejor valor nutritivo en los pastos, pues como se sabe, el contenido de MO está condicionado por los niveles de acidez en los suelos (Bernal, 1994; Burbano y Cadena, 2009; Tapia y Rivera, 2010). Por otro lado, la correlación entre MO y K, permitió que la planta asimile de mejor forma este mineral, ya que a menor $\mathrm{pH}$ existe mejor disponibilidad del elemento, el cual es indispensable para el adecuado desarrollo vegetativo y calidad nutritiva del pasto (Estrada, 2002). La correlación positiva del periodo de recuperación y la producción de biomasa, favorece la mayor producción de forraje verde en menos tiempo. A estos resultados también contribuyó el riego suministrado a los pastos, durante los periodos secos del año.

\section{CONCLUSIONES}

Lacorrelación positiva, reportada en este estudio entre el periodo de recuperación y producción de biomasa, corrobora que existieron adecuados valores de materia orgánica, $\mathrm{pH}$, densidad aparente, densidad real y porosidad total del suelo, las cuales permitieron obtener mayores producciones de forraje verde, en menor tiempo y con buenas características nutritivas.

El análisis integral de los componentes de un sistema pecuario, es importante para orientar los

Tabla 10. Correlaciones variables suelo -planta

\begin{tabular}{l|c|c|c|c|c|c}
\hline & $\begin{array}{c}\text { Proteína } \\
\text { del pasto } \\
\text { \% }\end{array}$ & $\mathbf{p H}$ & $\begin{array}{c}\text { MO } \\
\mathbf{\%}\end{array}$ & $\begin{array}{c}\mathbf{P} \\
(\mathbf{m g} / \mathbf{K g})\end{array}$ & $\begin{array}{c}\mathbf{K} \\
\text { (meq/100g) }\end{array}$ & $\begin{array}{c}\text { Periodo } \\
\text { Recuperación } \\
\text { (días) }\end{array}$ \\
\hline $\begin{array}{l}\text { Proteína del pasto \% } \\
\text { pH }\end{array}$ & $+1,0$ & & & & & \\
MO \% & $+1,0$ & $+1,0$ & & & & \\
P (mg/Kg) & $-1,0$ & $-1,0$ & $-1,0$ & & & \\
K (meq/100g) & $+1,0$ & $+1,0$ & $+1,0$ & $-1,0$ & & \\
$\begin{array}{l}\text { Periodo Recuperación } \\
\text { (días) }\end{array}$ & & $-1,0$ & $-1,0$ & $-1,0$ & $-1,0$ & \\
Biomasa $\left(\mathrm{Kg} / \mathrm{m}^{2}\right)$ & & $-1,0$ & $-1,0$ & $-1,0$ & $-1,0$ & $+1,0$ \\
\hline
\end{tabular}


programas de nutrición y alimentación del ganado lechero en el Trópico Alto de Nariño.

\section{BIBLIOGRAFÍA}

APRÁEZ, G.J., GÁLVEZ, C.A., TAPIA, C.E., JOJOA, L., LEÓN, J., ZAMBRANO, J., ZAMBRANO, H.R., OBANDO, V. y AUX, M.Y. 2012. Determinación de los factores edafoclimáticos que influyen en la producción y calidad del pasto Kikuyo (Pennisetum clandestinum) en condiciones de no intervención. Livestock Research for Rural Develomment. 24 (3).

BELTRÁN, R. y BENAVIDES, F. 2009. Determinación de los factores climáticos y edáficos que condicionan la producción y calidad nutritiva del pasto Saboya (Holcus lanatus L.) en suelos no intervenidos de las veredas Cualapud, Arvela y Santa Rosa del municipio de Guachucal -Nariño, con altitudes entre 3050 y 3300 msnm. Pasto, Colombia. Trabajo de Grado Zootecnista. Facultad de Ciencias Pecuarias. Universidad de Nariño. San Juan de Pasto. 111p.

BENÍTEZ, C. 1983. Los pastos en Cuba. Instituto de Ciencia Animal. La Habana, Cuba: Pueblo y Educación. 676 p.

BERNAL, J. 1994. Pastos y forrajes tropicales. Bogotá, Colombia. Tercera edición. Bogotá. 318 p.

BERNAL, E.J. 2003. Pastos y Forrajes tropicales, Producción y Manejo. Colombia: Cuarta edición. Ángel Agro, Ideagro. 421 p.

BURBANO, F. y CADENA, W. 2009. Determinación de las características edafoclimáticas que garantizan la producción y calidad nutritiva del pasto brasilero (Phalaris sp), en condiciones de no intervención, en un rango de altitud comprendida entre 3050 - 3300 m.s.n.m. en el municipio de Guachucal, departamento de Nariño. Tesis de grado Zootecnista, Facultad de Ciencias Pecuarias, Universidad de Nariño, San Juan de Pasto. $128 \mathrm{p}$.

CASTRO, R.E., MOJICA, R.J.E., LEÓN, J.M., PABÓN, M.L., CARULLA, .FJ.E., y CÁRDENAS, R.E.A. 2008. Productividad de pasturas y producción de leche bovina bajo pastoreo de gramínea y gramínea + Lotus uliginosus en Mosquera, Cundinamarca. Revista de Medicina Veterinaria y Zootecnia. 55:9-21.

CHARRY, J. 1987. Naturaleza y Propiedades Físicas de los Suelos. Editorial Universidad Nacional. $361 \mathrm{p}$.

DONALD, C. 2007. Fertilidad de suelos. México: Euned. 16 p.

DURÁN, R.F. 2003. Volvamos al Campo: Manual de Cultivos Orgánicos y Alelopatía. Bogotá - Colombia. Grupo Latino Ltda. 74 p.

ESTRADA, A.J. 2002. Pastos y Forrajes para el Trópico Colombiano. Ed. U. de Caldas, Manizales. 511 p.

GARCÉS, A.M., BERRIO, L., RUIZ, S., SERNA, J.G. y BUILES, A.F. 2004. Ensilaje como fuente de alimentación para el ganado. Revista Lasallista de Investigación. 1(1):66 - 71.

GAVILÁN, M. 2004. Tratado de cultivo sin suelo. España: Mundi-prensa. 122 p.

HERRERA, P. AMÉZQUITA, E., GUERRERO, L. y RESTREPO, L. 1989. Efecto de la labranza sobre algunas propiedades físicas del suelo andico. Suelos Ecuatoriales. 21(1):68 - 75.

INSTITUTO GEOGRÁFICO AGUSTÍN CODAZZI. (IGAC). 2003. Mapa de Suelos de Colombia (escala 1:500.000). Subd. de Agrología. 26 planchas y CD. Bogotá. 
INSTITUTO GEOGRÁFICO AGUSTÍN CODAZZI (IGAC). 2006. Métodos analíticos de laboratorio de suelos. 6 ed. IGAC, Bogotá. 513 p.

INSTITUTO AS RESEARCH GRASSLANDS DE NUEVA ZELANDA. 2010. Ficha técnica, línea de nutrición animal clima frío. Ray Grass Híbrido Perenne Teraploide Sterling. 1 p.

JARAMILLO, J. 2004. Ciencia del suelo. Notas de clase, Universidad del Valle, Facultad de Ingeniería. $69 \mathrm{p}$.

JOJOA, L. y SILVA, J. 2009. Determinación de los factores edafoclimáticos que afectan la productividad del pasto Kikuyo (Pennisetum clandestinum $\mathrm{H}$ ) en condiciones naturales en la zona rural del municipio de Ipiales y el municipio de Adana, Departamento de Nariño. Tesis de Grado Zootecnista, Facultad de Ciencias Pecuarias, Universidad de Nariño. 39 p.

LEÓN, J. y ZAMBRANO, D. 2008. Determinación de los factores edafoclimáticos que intervienen en la producción y calidad nutritiva del pasto Kikuyo (Pennisetum clandestinum Hoeschst) en condiciones de no intervención en el municipio de Pasto, Departamento de Nariño. Tesis de Grado Zootecnista, Facultad de Ciencias Pecuarias, Universidad de Nariño. 25 p.

MCDOWELL, L.R., VELÁSQUEZ, P.J. y VALLE, G. 1997. Minerales para rumiantes en pastoreo en regiones tropicales. Tercera Edición. Centro de Agricultura Tropical. Universidad de Florida. Gainesville, Florida. 84 p.

MERA, R. y RUALES, H. 2007. Evaluación de la adaptación del trébol pata de pájaro (Lotus corniculatus) asociado con Kikuyo (Pennisetum clandestinum) bajo dos sistemas de reproducción y diferentes densidades de siembra en el municipio Pasto - Nariño. Tesis de Grado Zootecnista,
Facultad de Ciencias Pecuarias, Universidad de Nariño. Pasto. 37 p.

MINISTERIO DE AGRICULTURA Y DESARROLLO RURAL (MADR). 2008. Secretaría de Agricultura y Medio Ambiente. Consolidado Agropecuario. En: www.minagricultura.gov. co/; consulta: enero, 2014.

MILA, A. 2001. Suelos, pastos y forrajes: Producción y manejo. Bogotá: UNISUR. 88 p.

MOLINA, C., OVALLE, L. y GÁRNICA, J. 1982. Avances de la investigación en pastizales en las zonas altas de los Andes. Mérida. FONAIAP DIVULGA No. 07. En: http://sian.inia.gob.ve/repositorio/revistas_tec/FonaiapDivulga/fd07/ texto/avances.htm; consulta: enero, 2014.

NATIONAL RESEARCH COUNCIL (NRC). 1989. Nutrient Requirements of Dairy Cattle. 6th rev. ed. National Academy Press. Washington, D. C. 157 p.

PARRA, M.A. 2003. Los suelos y la fertilización del olivar cultivado en zonas calcáreas. Andalucía: Mundiprensa. $45 \mathrm{p}$.

PIRELA, M.F. 2007. Valor nutritivo de los pastos tropicales. Instituto Nacional de Investigaciones Agrícolas. En: http://www.avpa.ula.ve/docuPDFs/libros_online/manualganaderia/seccion3/articulo6-s3.pdf; consulta: enero, 2014.

PROGRAMA DE MEJORAMIENTO GENÉTICO-MEGALAC. 2009. Convenio Universidad de Nariño, Colácteos y Ministerio de Agricultura y Desarrollo Rural. En: http://promegalac.udenar.edu.co; consulta: diciembre, 2013.

RODAS, A. 2007. Producción Verde - Especies forrajeras de clima frío. En: Seminario de Gestión de Empresas Ganaderas. (10: 2007 
Pasto). Memorias del I Seminario de Gestión de Empresas Ganaderas. Pasto: Universidad de Nariño. 58 p.

SALAMANCA, S.R. 1986. Pastos y Forrajes Producción y Manejo. Bogotá - Colombia: Universidad Santo Tomás. 124 - 125 p.

SÁNCHEZ, J.M. 2000. Hipomagnesemia. Un Desbalance Metabólico Subestimado en Nuestros Hatos de Ganado Lechero. En Costa Rica. Nutrición Animal Tropical. 6(1): 75 - 95.

SÁNCHEZ, J.M. 2007. Utilización eficiente de las pasturas tropicales en la alimentación del ganado lechero. p24. XI Seminario de Pastos y Forrajes en Sistemas de Producción Animal. 30 p.

SORIANO, S.M y PONS, M.V. 2004. Prácticas de Edafología y Climatología. México D. F: Alfaomega Grupo Editor. 33:35 - 38.

TAPIA, C.E y RIVERA, C.C. 2010. Determinación de los factores climáticos y edáficos que intervienen en la producción y calidad nutritiva del pasto kikuyo (Pennisetum clandestinum Hoeschst) en condiciones de no intervención en el municipio de Guachucal, departamento de Nariño. Pasto - Colombia. Trabajo de grado Zootecnista. Facultad de Ciencias Pecuarias. Universidad de Nariño. San Juan de Pasto. 99 p.

THOMPSON, L. 1988. Los suelos y su fertilidad. $4^{\mathrm{a}}$ edición, Texas: Reverté. 80 p.

UNIGARRO, S.A., INSUASTY, B.R. y CHAVES, J.G. 2009. Manual de prácticas de laboratorios suelos generales, Primera Edición, Editorial Universitaria, Universidad de Nariño. 25 - 105 p.

URBANO P. 1992. Tratado de Fitotecnia General. Zaragoza: Mundi-prensa. 544 p.
VILORIA, J. 2007. Economía del Departamento de Nariño: ruralidad y aislamiento geográfico. Banco de la República, Centro de Estudios Económicos Regionales (CEER) - Cartagena Colombia. $87 \mathrm{p}$.

WEISS, W.P. 2000. Update on Mineral Requirements for Dairy Cattle. IN: Curso de Nutrición de Ganado Lechero. Balsa, Atenas. Costa Rica. $1-10 \mathrm{p}$. 